\title{
ON GENERALIZED THETA SERIES LIFTINGS
}

\author{
MIN HO LEE \\ (Received 15 February 1995) \\ Communicated by R. Howlett
}

\begin{abstract}
We generalize dual reductive pairs by using reductive groups that are not necessarily subgroups of symplectic groups and construct the corresponding theta-series liftings for certain types of automorphic forms. We also discuss connections of such generalized theta-series liftings with families of abelian varieties parametrized by an arithmetic variety.
\end{abstract}

1991 Mathematics subject classification (Amer. Math. Soc.): primary 11F27; secondary 11F70.

\section{Introduction}

Let $W$ be a finite-dimensional vector space over a number field $k$ equipped with a nondegenerate alternating bilinear form, and let $\left(U_{1}, U_{2}\right)$ be a dual reductive pair in the symplectic group $S p(W)$ in the sense of Howe (see [4]). Thus $U_{1}$ and $U_{2}$ are subgroups of $S p(W)$ that are mutual centralizers. Given such a dual reductive pair, the theta-series lifting that associates an automorphic form on $U_{1}$ (respectively $U_{2}$ ) to a cusp form on $U_{2}$ (respectively $U_{1}$ ) can be determined by using the Weil representation. Thus dual reductive pairs play an important role in constructing automorphic forms and representations of reductive groups. They also have been useful in other applications such as the construction of automorphic $L$-functions. Despite their usefulness, one disadvantage of dealing with dual reductive pairs is the fact that the groups involved must be subgroups of a given symplectic group. In this paper, we attempt to extend the concept of dual reductive pairs to certain reductive groups that are not necessarily subgroups of a symplectic group.

Let $G$ and $G^{\prime}$ be reductive groups defined over $k$, and let $\rho: G \rightarrow G^{\prime}$ be a homomorphism defined over $k$. Let $\mathbb{A}$ (respectively $\mathbb{A}_{f}$ ) be the ring of adeles (respectively finite adeles) of $k$. Let $K$ (respectively $K^{\prime}$ ) be a maximal compact subgroup

(C) 1997 Australian Mathematical Society $0263-6115 / 97 \$ A 2.00+0.00$ 
of $G\left(\mathbb{A}_{f}\right)$ (respectively $G^{\prime}\left(\mathbb{A}_{f}\right)$ ) such that $\rho(K) \subset K^{\prime}$. Then a mixed automorphic form on $G(\mathbb{A})$ of type $\left(\rho, K, K^{\prime}\right)$ is a function on $G(\mathbb{A})$ of the form $f \cdot\left(f^{\prime} \circ \rho\right)$, where $f$ (respectively $f^{\prime}$ ) is an automorphic form on $G(\mathbb{A})$ ) (respectively $G^{\prime}(\mathbb{A})$ ). It is known that holomorphic mixed automorphic forms of certain types arise naturally as holomorphic forms of the highest degree on certain families of abelian varieties parametrized by an arithmetic variety (see for example $[6,9,12,13])$. A generalized automorphic form of type $\left(\rho, K, K^{\prime}\right)$ is a function on $G(\mathbb{A})$ of the form $f^{\prime} \circ \rho$ for some automorphic form $f^{\prime}$ on $G^{\prime}(\mathbb{A})$.

The purpose of this paper is to generalize dual reductive pairs and construct theta series-liftings for mixed automorphic forms and generalized automorphic forms. In particular such theta-series liftings will provide correspondences between automorphic forms on reductive groups that are not necessarily subgroups of symplectic groups. We also discuss geometric applications of such correspondences in connection with certain families of abelian varieties.

\section{Automorphic forms}

In this section we review the definition of automorphic forms (see for example [1]) on a reductive group over a number field and introduce special types of automorphic forms associated to homomorphisms of reductive groups. Let $k$ be a number field, and let $\mathbb{A}$ (respectively $\mathbb{A}_{f}$ ) be the ring of adeles (respectively finite adeles) of $k$. For each place $v$ of $k$, we denote by $k_{v}$ the completion of $k$ at $v$. Let $G$ be a reductive group defined over $k$, and let $K$ be a maximal compact subgroup of $G(\mathbb{A})$. We set $G_{\infty}=\prod_{v \in V_{\infty}} G\left(k_{v}\right)$ where $V_{\infty}$ is the set of infinite places of $k$. Let $g_{\infty}$ be the Lie algebra of $G_{\infty}$, and let $Z\left(\mathfrak{g}_{\infty}\right)$ be the center of the universal enveloping algebra $U\left(\mathfrak{g}_{\infty}\right)$ of $\mathfrak{g}_{\infty}$.

DEFINITION 2.1. A smooth function $f$ on $G(\mathbb{A})$ is an automorphic form for $K$ if it satisfies the following conditions:

(i) $f(\gamma x)=f(x)$ for all $\gamma \in G(k)$ and $x \in G(\mathbb{A})$.

(ii) $f$ is $K$-finite.

(iii) $f$ is $Z\left(\mathfrak{g}_{\infty}\right)$-finite.

(iv) For each $y \in G\left(\mathbb{A}_{f}\right)$ the function $x \mapsto f(x y)$ on $G_{\infty}$ is slowly increasing.

The function $f$ is a cusp form if in addition it is cuspidal, that is,

$$
\int_{N(k) \backslash N(\mathbb{A})} f(n x) d n=0
$$

for all $x \in G(\mathbb{A})$, where $N$ is the unipotent radical of any proper parabolic $k$-subgroup $P$ of $G$ (see for example [1] for details). 
Now we want to introduce special types of automorphic forms. Let $G^{\prime}$ be another reductive group over $k$, and let $K^{\prime}$ be a maximal compact subgroup of $G^{\prime}(\mathbb{A})$. Let $\rho: G \rightarrow G^{\prime}$ be a homomorphism defined over $k$ such that $\rho(K) \subset K^{\prime}$.

DEFINITION 2.2. A smooth function $f$ on $G(\mathbb{A})$ is a generalized automorphic (respectively cusp) form of type ( $\left.\rho, K, K^{\prime}\right)$ if there is an automorphic (respectively cusp) form $f^{\prime}$ on $G^{\prime}(\mathbb{A})$ for $K^{\prime}$ such that $f=f^{\prime} \circ \rho$.

DEFINITION 2.3. A smooth function $f$ on $G(\mathbb{A})$ is a mixed automorphic form of type $\left(\rho, K, K^{\prime}\right)$ if there are an automorphic form $f_{1}$ on $G(\mathbb{A})$ for $K$ and an automorphic form $f_{2}$ on $G^{\prime}(\mathbb{A})$ for $K^{\prime}$ such that $f=f_{1} \cdot\left(f_{2} \circ \rho\right)$.

REMARK 2.4. The word 'generalized' in Definition 2.2 might not be appropriate in some sense. The function $f$ becomes an automorphic (respectively cusp) form in the sense of Definition 2.1 if $G$ coincides with $G^{\prime}$ and $\rho$ is the identity homomorphism. In this sense it is a generalized automorphic (respectively cusp) form. However, a generalized automorphic form in the sense of Definition 2.2 is also a special case of an automorphic form due to Theorem 2.9 below. We used this term because of an obvious analogy with generalized automorphic forms considered by W. Hoyt and P. Stiller in the elliptic modular case (see for example [2, p. 31], [16, $\$ 3$ bis]).

REMARK 2.5. In the case of holomorphic automorphic forms, certain mixed automorphic forms of the type given in Definition 2.3 occur naturally as holomorphic forms of the highest degree on certain families of abelian varieties parametrized by an arithmetic variety (see for example $[6,9,11,12,13]$ ).

LEMMA 2.6. Let $f$ be a $K$-finite function on $G(\mathbb{A})$ and $f^{\prime}$ a $K^{\prime}$-finite function on $G^{\prime}(\mathbb{A})$. Then both $f^{\prime} \circ \rho$ and $f_{1} \cdot\left(f^{\prime} \circ \rho\right)$ are $K$-finite functions on $G(\mathbb{A})$.

PROOF. For each $k \in K$, let $r(k) f$ (respectively $r(k) f^{\prime}$ ) denote the function on $G(\mathbb{A})$ (respectively $G^{\prime}(\mathbb{A})$ ) given by

$$
r(k) f(x)=f(x k) \quad\left(\text { respectively } r(k) f^{\prime}\left(x^{\prime}\right)=f^{\prime}\left(x^{\prime} k\right)\right) .
$$

Since $f$ is $K$-finite and $f^{\prime}$ is $K^{\prime}$-finite, there are elements $k_{1}, \ldots, k_{\mu} \in K$ and $k_{1}^{\prime}, \ldots, k_{v}^{\prime} \in K^{\prime}$ such that for each $k \in K$ and $k^{\prime} \in K^{\prime}$

$$
\begin{aligned}
r(k)\left(f(x) \cdot f^{\prime}(y)\right) & =f(x k) \cdot f^{\prime}\left(y k^{\prime}\right) \\
& =\sum_{i=1}^{\mu} \sum_{j=1}^{\nu} a_{i j}\left(k, k^{\prime}\right) r\left(k_{i}\right) f(x) \cdot r\left(k_{j}^{\prime}\right) f^{\prime}(y)
\end{aligned}
$$


for some constants $a_{i j}\left(k, k^{\prime}\right)$ and for all $x \in G(\mathbb{A})$ and $y \in \mathbb{G}^{\prime}(\mathbb{A})$. Thus for each $k \in K$ we have

$$
\begin{aligned}
r(k)\left(f \cdot\left(f^{\prime} \circ \rho\right)\right)(x) & \left.=f(x k) \cdot f^{\prime}(\rho(x) \rho(k))\right) \\
& =\sum_{i=1}^{\mu} \sum_{j=1}^{\nu} a_{i j}(k, \rho(k)) r\left(k_{i}\right) f(x) \cdot r\left(k_{j}^{\prime}\right) f^{\prime}(\rho(x)) .
\end{aligned}
$$

Therefore the set $\left\{r(k) \cdot\left(f \cdot\left(f^{\prime} \circ \rho\right)\right) \mid k \in K\right\}$ is finite-dimensional; hence $f \cdot\left(f^{\prime} \circ \rho\right)$ is $K$-finite. If $f$ is the constant function 1 , then $f \cdot\left(f^{\prime} \circ \rho\right)=f^{\prime} \circ \rho$, therefore the $K$-finiteness of $f^{\prime} \circ \rho$ also follows.

LEMMA 2.7. Let $f$ be a $Z\left(\mathfrak{g}_{\infty}\right)$-finite function on $G(\mathbb{A})$ and $f^{\prime}$ a $Z\left(g_{\infty}^{\prime}\right)$-finite function on $G^{\prime}(\mathbb{A})$. Then both $f^{\prime} \circ \rho$ and $f \cdot\left(f^{\prime} \circ \rho\right)$ are $Z\left(g_{\infty}\right)$-finite functions on $G(\mathbb{A})$.

PROOF. When $f$ is considered as a function on $G_{\infty}$, an element $Y \in Z\left(g_{\infty}\right)$ operates on $f$ by

$$
(Y \cdot f)(g)=\left.\frac{d}{d t} f((\exp t Y) g)\right|_{t=0}
$$

for $g \in G_{\infty}$. Given $Y \in Z\left(\mathfrak{g}_{\infty}\right)$, we have

$$
\begin{aligned}
Y \cdot(f & \left.\cdot\left(f^{\prime} \circ \rho\right)\right)(g)=\left.\frac{d}{d t}\left(f \cdot\left(f^{\prime} \circ \rho\right)\right)((\exp t Y) g)\right|_{t=0} \\
& =\left[\frac{d}{d t} f((\exp t Y) g)\right]_{t=0} \cdot f^{\prime}(\rho(g))+f(g) \cdot\left[\frac{d}{d t} f^{\prime}(\rho((\exp t Y) g))\right]_{t=0} \\
& =\left[\frac{d}{d t} f((\exp t Y) g)\right]_{t=0} \cdot f^{\prime}(\rho(g))+f(g) \cdot\left[\frac{d}{d t} f^{\prime}((\exp t d \rho Y) \rho(g))\right]_{t=0} .
\end{aligned}
$$

Thus the $Z\left(\mathfrak{g}_{\infty}\right)$-finiteness of $f \cdot\left(f^{\prime} \circ \rho\right)$ follows from the fact that $f$ is $Z\left(\mathfrak{g}_{\infty}\right)$-finite and $f^{\prime}$ is $Z\left(\mathfrak{g}_{\infty}^{\prime}\right)$-finite, and the $Z\left(\mathfrak{g}_{\infty}\right)$-finiteness of $f^{\prime} \circ \rho$ is also obtained by taking $f \equiv 1$.

LEMMA 2.8. Let the functions $f_{\infty}$ on $G_{\infty}$ and $f_{\infty}^{\prime}$ be slowly increasing. Then both $f_{\infty}^{\prime} \circ \rho$ and $f_{\infty} \cdot\left(f_{\infty}^{\prime} \circ \rho\right)$ are slowly increasing functions on $G_{\infty}$.

PROOF. Recall that a function $f$ on $G_{\infty}$ is slowly increasing if there is a constant $C$ such that $|f(x)| \leq C\|x\|_{\sigma}^{n}$ for all $x \in G_{\infty}$, where $\sigma: G_{\infty} \rightarrow G L(E)$ is a finitedimensional complex representation with finite kernel and image closed in End $(E)$ and $\|x\|=\left(\operatorname{tr} \sigma(x)^{*} \cdot \sigma(x)\right)^{1 / 2}$ (see [1]). Since $f_{\infty}$ and $f_{\infty}^{\prime}$ are slowly increasing, we have

$$
\left|\left(f_{\infty} \cdot\left(f_{\infty}^{\prime} \circ \rho\right)\right)(g)\right|=\left|f_{\infty}(g) \cdot f_{\infty}^{\prime}(\rho(g))\right| \leq C_{1}\|g\|_{\alpha}^{m_{1}} \cdot C_{2}\|\rho(g)\|_{\beta}^{m_{2}}
$$


for some constants $C_{1}, C_{2}$, positive integers $m_{1}, m_{2}$, and representations $\alpha: G \rightarrow$ $G L(W), \beta: G \rightarrow G L\left(W^{\prime}\right)$. However, we have $\|\rho(g)\|_{\beta}=\|g\|_{\beta \circ \rho} \leq C_{3}\|g\|_{\alpha}^{m_{3}}$ for some constant $C_{3}$ and positive integer $m_{3}$. Thus we have

$$
\left|\left(f_{\infty} \cdot\left(f_{\infty}^{\prime} \circ \rho\right)\right)(g)\right| \leq C_{1} C_{2} C_{3}\|g\|_{\alpha}^{m_{1}+m_{3}},
$$

and therefore $f_{\infty} \cdot\left(f_{\infty}^{\prime} \circ \rho\right)$ is slowly increasing. We also see that $f_{\infty}^{\prime} \circ \rho$ is slowly increasing by taking $f_{\infty} \equiv 1$.

THEOREM 2.9. Both a generalized automorphic form and a mixed automorphic form of type $\left(\rho, K, K^{\prime}\right)$ are automorphic forms for $K$ in the sense of Definition 2.1.

PROOF. Let $f_{1}$ be a generalized automorphic form and $f_{2}$ a mixed automorphic form on $G(\mathrm{~A})$ of the form

$$
f_{1}=f^{\prime} \circ \rho, \quad f_{2}=f \cdot\left(f^{\prime} \circ \rho\right),
$$

where $f$ (respectively $f^{\prime}$ ) is an automorphic form for $K$ (respectively $K^{\prime}$ ) on $G(\mathbb{A})$ (respectively $G^{\prime}(\mathbb{A})$ ). Then for each $g \in G(k)$ we have

$$
\begin{aligned}
& f_{1}(g x)=f^{\prime}(\rho(g x))=f^{\prime}(\rho(g) \rho(x))=f^{\prime}(\rho(x)), \\
& f_{2}(g x)=f(g x) \cdot f^{\prime}(\rho(g) \rho(x))=f(x) \cdot f^{\prime}(\rho(x))=f(x)
\end{aligned}
$$

for all $x \in G(\mathbb{A})$, since $\rho(G(k)) \subset G^{\prime}(k)$; hence both $f_{1}$ and $f_{2}$ satisfy the condition (i) of Definition 2.1. They also satisfy the conditions (ii), (iii), and (iv) of Definition 2.1 by Lemmas $2.6,2.7$, and 2.8 , respectively. Hence it follows that both $f_{1}$ and $f_{2}$ are automorphic forms.

\section{Generalized theta-series liftings}

In this section, we first review theta-series liftings associated to dual reductive pairs, and then extend such liftings to certain generalized dual reductive pairs. Let $W$ be a symplectic space over a number field $k$, that is, a vector space over $k$ equipped with a nondegenerate alternating bilinear form $\langle$,$\rangle . Then the Heisenberg group$ $\mathscr{H}(W)$ associated to $W$ is a non-trivial central extension of $W$ by $k$ consisting of pairs $\{(w, t) \mid w \in W, t \in k\}$ with its multiplication law given by

$$
\left(w_{1}, t_{1}\right) \cdot\left(w_{2}, t_{2}\right)=\left(w_{1}+w_{2}, t_{1}+t_{2}+\left\langle w_{1}, w_{2}\right\rangle / 2\right) .
$$

We fix a complete polarization $W=W_{1} \oplus W_{2}$ of $W$, where $W_{1}$ and $W_{2}$ are maximal totally isotropic subspaces of $W$. Let $\mathscr{S}\left(W_{1}(A)\right)$ be the space of Schwartz-Bruhat 
functions on $W_{1}(\mathbb{A})$. Given a character $\psi: \mathbb{A} / k \rightarrow \mathbb{C}^{\times}$we can construct a representation $\sigma_{\psi}$ of $\mathscr{H}(W)(\mathbb{A})$ on $\mathscr{S}\left(W_{1}(\mathbb{A})\right)$ by

$$
\begin{aligned}
& \sigma_{\psi}\left(w_{1}\right) f(x)=f\left(x+w_{1}\right) \quad \text { for all } \quad x, w_{1} \in W_{1}(\mathbb{A}), \\
& \sigma_{\psi}\left(w_{2}\right) f(x)=\psi\left(\left\langle x, w_{2}\right\rangle\right) f(x) \quad \text { for all } \quad x \in W_{1}(\mathbb{A}), w_{2} \in W_{2}(\mathbb{A}) \text {, } \\
& \sigma_{\psi}(t) f(x)=\psi(t) f(x) \quad \text { for all } t \in \mathbb{A}, x \in W_{1}(\mathbb{A}) \text {. }
\end{aligned}
$$

We define a linear functional $\Theta$, known as the theta-functional, on $\mathscr{S}\left(W_{1}(\mathbb{A})\right)$ by $\Theta(\varphi)=\sum_{x \in W_{1}(k)} \varphi(x)$ for all $\varphi \in \mathscr{S}\left(W_{1}(\mathbb{A})\right)$. Then it is known that the series defining $\Theta(\varphi)$ converges absolutely and the map $\varphi \mapsto \Theta(\varphi)$ defines an $\mathscr{H}(W)(k)$-invariant linear form on $\mathscr{S}\left(W_{1}(\mathbb{A})\right)$ (cf. [17]). The adelic symplectic group $S p(W)(\mathbb{A})=$ $S p(W,\langle\rangle),(\mathbb{A})$ operates on the adelic Heisenberg group $\mathscr{H}(W)(\mathbb{A})$ by $g \cdot(w, t)=$ $(g w, t)$. This operation is trivial on the center of $\mathscr{H}(W)(\mathbb{A})$ and therefore by the Stone-von Neumann theorem there is an operator $\omega_{\psi}$ on $\mathscr{S}\left(W_{1}(A)\right)$ unique up to a constant factor such that

$$
\sigma_{\psi}(g w, t) \cdot \omega_{\psi}(g)=\omega_{\psi}(g) \cdot \sigma_{\psi}(w, t)
$$

for all $(w, t) \in \mathscr{H}(W)(\mathbb{A})$. Now we define the metaplectic group $\widetilde{S p}(W)(\mathbb{A})$ to be the set of pairs $\left(g, \omega_{\psi}(g)\right)$ satisfying the above relation. Then we have the exact sequence

$$
0 \rightarrow \mathbb{C}^{\times} \rightarrow \widetilde{S p_{p}}(W)(\mathbb{A}) \rightarrow S p(W)(\mathbb{A}) \rightarrow 0 .
$$

Let $\left(G^{\prime}, H^{\prime}\right)$ with $G^{\prime}, H^{\prime} \subset S p(W)$ be a dual reductive pair in the symplectic group $S p(W)=S p(W,\langle\rangle$,$) (see for example [3,4,5,14]$ ). Thus $G^{\prime}$ and $H^{\prime}$ are reductive subgroups of $S p(W)$ and $G^{\prime}$ (respectively $H^{\prime}$ ) is the centralizer of $H^{\prime}$ (respectively $G^{\prime}$ ) in $S p(W)$. Let $\tilde{G}^{\prime}(\mathbb{A})$ (respectively $\tilde{H}^{\prime}(\mathbb{A})$ ) be the inverse image of $G^{\prime}(\mathbb{A})$ (respectively $\left.H^{\prime}(\mathbb{A})\right)$ in $\widetilde{S p}(W)(\mathbb{A})$. Then given an element $\varphi \in \mathscr{S}\left(W_{1}(\mathbb{A})\right)$ the theta-kernel for the pair $\left(G^{\prime}, H^{\prime}\right)$ is the map $\theta_{\varphi}: \tilde{G}^{\prime}(\mathbb{A}) \times \tilde{H}^{\prime}(\mathbb{A}) \rightarrow \mathbb{C}$ given by

$$
\theta_{\varphi}\left(g^{\prime}, h^{\prime}\right)=\Theta\left(\omega_{\psi}\left(g^{\prime}\right) \omega_{\psi}\left(h^{\prime}\right)(\varphi)\right) .
$$

For each cusp from $f^{\prime}$ on $\tilde{G}^{\prime}(\mathbb{A})$ the theta-kernel $\theta_{\varphi}$ determines the map $\theta_{\varphi}\left(f^{\prime}\right)$ : $\tilde{H}^{\prime}(\mathbb{A}) \rightarrow \mathbb{C}$ given by

$$
\theta_{\varphi}\left(f^{\prime}\right)\left(h^{\prime}\right)=\int_{G^{\prime}(k) \backslash \hat{G}^{\prime}(\mathbb{A})} \theta_{\varphi}\left(g^{\prime}, h^{\prime}\right) f^{\prime}\left(g^{\prime}\right) d g^{\prime} .
$$

Let $\tilde{K}_{W}$ be a maximal vompact subgroup of $\widetilde{S p}(W(\mathbb{A}))$, and set

$$
\tilde{K}^{\prime}=\tilde{K}_{W} \cap \tilde{G}^{\prime}(\mathbb{A}), \quad \tilde{K}_{H}^{\prime}=\tilde{K}_{W} \cap \tilde{H}^{\prime}(\mathbb{A}) .
$$

Then $K^{\prime}$ and $K_{H}^{\prime}$ are maximal compact subgroups of $\tilde{G}^{\prime}(\mathbb{A})$ and $\tilde{H}^{\prime}(\mathbb{A})$ respectively. 
PROPOSITION 3.1. If $f$ is a cusp form on $\tilde{G}^{\prime}(\mathbb{A})$ and if $\varphi$ is $K_{H}^{\prime}$-finite, then $\theta_{\varphi}(f)$ is an automorphic form on $\tilde{H}^{\prime}(\mathbb{A})$.

ProOF. See [5, Proposition 2.2].

In order to generalize the result of Proposition 3.1, let $\tilde{G}$ and $\tilde{H}$ be reductive groups over $k$ such that there are homomorphisms $\tilde{\rho}: \tilde{G} \rightarrow \tilde{G}^{\prime}$ and $\tilde{\rho}_{H}: \tilde{H} \rightarrow \tilde{H}^{\prime}$ defined over $k$. Thus the pair $(\tilde{G}, \tilde{H})$ can be regarded as a generalized dual reductive pair. Let $\tilde{K}$ and $\tilde{K}_{H}$ be maximal subgroups of $\tilde{G}(\mathbb{A})$ and $\tilde{H}(\mathbb{A})$, respectively, such that $\tilde{\rho}(\tilde{K}) \subset \tilde{K}^{\prime}$ and $\tilde{\rho}_{H}\left(\tilde{K}_{H}\right) \subset \tilde{K}_{H}^{\prime}$.

DEFINITION 3.2. Let $f$ be a generalized cusp form on $\tilde{G}(\mathbb{A})$ of type $\left(\tilde{\rho}, \tilde{K}, \tilde{K}^{\prime}\right)$, so that $f=f \circ \tilde{\rho}$ for some cusp form $f^{\prime}$ on $\tilde{G}(\mathrm{~A})$. Then for each $\varphi \in \mathscr{S}\left(W_{1}(\mathbb{A})\right)$ we define the $\operatorname{map} \theta_{\varphi}^{\tilde{\rho}, \tilde{\rho}_{H}}(f): \tilde{H}(\mathbb{A}) \rightarrow \mathbb{C}$ by

$$
\theta_{\varphi}^{\tilde{\rho}, \tilde{\rho}_{H}}(f)(h)=\theta_{\varphi}\left(f^{\prime}\right)\left(\tilde{\rho}_{H}(h)\right)
$$

for all $h \in \tilde{H}(\mathbb{A})$.

THEOREM 3.3. Let $f$ be a generalized cusp form on $\tilde{G}(\mathbb{A})$ of type $\left(\tilde{\rho}, \tilde{K}, \tilde{K}^{\prime}\right)$. If $\varphi$ is $\tilde{K}_{H}^{\prime}$-finite, then $\theta_{\varphi}^{\tilde{\rho}, \tilde{\rho}_{H}}(f)$ is generalized automorphic form of type $\left(\tilde{\rho}_{H}, \tilde{K}_{H}, \tilde{K}_{H}^{\prime}\right)$.

PRoOF. Let $f=f^{\prime} \circ \tilde{\rho}$ for some cusp form $f^{\prime}$ on $\tilde{G}(\mathbb{A})$. Since $\varphi$ is $\tilde{K}_{H}^{\prime}$-finite, it follows from Proposition 3.1 that $\theta_{\varphi}\left(f^{\prime}\right)$ is an automorphic form on $\tilde{H}^{\prime}(\mathbb{A})$. Since $\theta_{\varphi}^{\tilde{\rho}, \bar{\rho}_{H}}(f)=\theta_{\varphi}\left(f^{\prime}\right) \circ \tilde{\rho}_{H}$, and $\tilde{\rho}_{H}\left(\tilde{K}_{H}\right) \subset \tilde{K}_{H}^{\prime}$, by Definition $2.2 \theta_{\varphi}^{\tilde{\rho}, \tilde{\rho}_{H}}(f)$ is a generalized automorphic form of type $\left(\tilde{\rho}_{H}, \tilde{K}_{H}, \tilde{K}_{H}^{\prime}\right)$.

REMARK 3.4. From Theorem 2.9 it follows that the function $f$ (respectively $\left.\theta_{\varphi}^{\bar{\rho}, \tilde{\rho}_{H}}(f)\right)$ in Theorem 3.3 is an automorphic form for $\tilde{K}$ (respectively $\tilde{K}^{\prime}$ ). Thus Theorem 3.3 can be used to establish correspondences between automorphic forms on reductive groups that are not necessarily subgroups of symplectic groups.

\section{Theta-series liftings for mixed automorhic forms}

In order to discuss theta-series liftings for mixed automorphic forms, in this section we consider a pair of dual reductive pairs. Let $W$ and $V$ by symplectic spaces over a number field $k$. Let $\left(G^{\prime}, H^{\prime}\right)$ be a dual reductive pair in $S p(W)$, and let $(G, H)$ be also a reductive pair in $S p(V)$ such that there are homomorphisms $\rho: G \rightarrow G^{\prime}$ and $\rho_{H}: H \rightarrow H^{\prime}$. 
Let $\tilde{G}^{\prime}(\mathbb{A})$ (respectively $\tilde{H}^{\prime}(\mathbb{A})$ ) be the inverse image of $G^{\prime}(\mathbb{A})$ (respectively $H^{\prime}(\mathbb{A})$ ) in $\widetilde{S p}(W)(\mathbb{A})$ as in Section 3. Similarly, let $\tilde{G}^{\prime}(\mathbb{A})$ (respectively $\left.\tilde{H}^{\prime}(\mathbb{A})\right)$ be the inverse image of $G^{\prime}(\mathbb{A})$ (respectively $H^{\prime}(\mathbb{A})$ ) in $\widetilde{S p}(V)(\mathbb{A})$. Using the exact sequences

$$
\begin{gathered}
0 \rightarrow \mathbb{C}^{\times} \rightarrow \widetilde{S p}(W)(\mathbb{A}) \rightarrow S p(W)(\mathbb{A}) \rightarrow 0, \\
0 \rightarrow \mathbb{C}^{\times} \rightarrow \widetilde{S p}(V)(\mathbb{A}) \rightarrow S p(V)(\mathbb{A}) \rightarrow 0,
\end{gathered}
$$

we can extend homomorphisms $\rho: G(\mathbb{A}) \rightarrow G^{\prime}(\mathbb{A})$ and $\rho_{H}: H(\mathbb{A}) \rightarrow H^{\prime}(\mathbb{A})$ to homomorphisms $\tilde{\rho}: \tilde{G}(\mathbb{A}) \rightarrow \tilde{G}^{\prime}(\mathbb{A})$ and $\tilde{\rho}_{H}: \tilde{H}(\mathbb{A}) \rightarrow \tilde{H}^{\prime}(\mathbb{A})$, respectively. Let $\tilde{K}^{\prime}$ (respectively $\tilde{K}_{H}^{\prime}$ ) be the maximal compact subgroup of $\tilde{G}^{\prime}(\mathbb{A})$ (respectively $\tilde{H}^{\prime}(\mathbb{A})$ ) associated to a maximal compact subgroup $\tilde{K}_{W}$ of $\widetilde{S p}(W)(\mathbb{A})$. Similarly, let $\tilde{K}$ (respectively $\tilde{K}_{H}$ ) be the maximal compact subgroup of $\tilde{G}(\mathbb{A})$ (respectively $\tilde{H}(\mathbb{A})$ ) associated to a maximal compact subgroup $\tilde{K}_{V}$ of $\widetilde{S p}(V)(\mathbb{A})$ such that $\tilde{\rho}(\tilde{K}) \subset \tilde{K}^{\prime}$ (respectively $\left.\tilde{\rho}_{H}\left(\tilde{K}_{H}\right) \subset \tilde{K}_{H}^{\prime}\right)$.

As in the case of $W$ described in Section 3, given a complete polarization $V=$ $V_{1} \oplus V_{2}$ of $V$, we can define $\theta_{\varphi_{V}}$, where $\varphi_{V}$ is an element of the space $\mathscr{S}\left(V_{1}(\mathbb{A})\right)$ of Schwartz-Bruhat functions on $V_{1}(\mathbb{A})$.

DEFINITION 4.1. Let $f$ be a mixed cusp form on $\tilde{G}(\mathbb{A})$ of type $\left(\tilde{\rho}, \tilde{K}, \tilde{K}^{\prime}\right)$, so that $f=f_{1} \cdot\left(f_{2} \circ \tilde{\rho}\right)$ for some forms $f$ on $\tilde{G}(\mathbb{A})$ and $f_{2}$ on $\tilde{G}^{\prime}(\mathbb{A})$. Then for each $\varphi_{V} \in \mathscr{S}\left(V_{1}(\mathbb{A})\right)$ and $\varphi \in \mathscr{S}\left(W_{1}(\mathbb{A})\right)$ we define the map $\theta_{\varphi_{v}, \varphi}^{\tilde{\rho}, \tilde{\rho}_{H}}(f): \tilde{H}(\mathbb{A}) \rightarrow \mathbb{C}$ by

$$
\theta_{\varphi_{\nu}, \varphi}^{\tilde{\rho}, \tilde{\rho}_{H}}(f)(h)=\theta_{\varphi_{V}}\left(f_{1}\right)(h) \cdot \theta_{\varphi}\left(f_{2}\right)\left(\tilde{\rho}_{H}(h)\right)
$$

for all $h \in \tilde{H}(\mathbb{A})$.

THEOREM 4.2. Let $\varphi_{V} \in \mathscr{S}\left(V_{1}(\mathbb{A})\right)$ be $\tilde{K}_{H}$-finite and let $\varphi \in \mathscr{S}\left(W_{1}(\mathbb{A})\right)$ be $\tilde{K}_{H}^{\prime}$ finite. If $f$ is a mixed cusp from on $\tilde{G}$ of type $\left(\tilde{\rho}, \tilde{K}, \tilde{K}^{\prime}\right)$, then the function $\theta_{\varphi_{V}, \varphi}^{\tilde{\rho}, \tilde{\rho}_{H}}(f)$ is a mixed automorphic from on $\tilde{H}(\mathbb{A})$ of type $\left(\tilde{\rho}_{H}, \tilde{K}_{H}, \tilde{K}_{H}^{\prime}\right)$.

PROOF. Since $\varphi_{V} \in \mathscr{S}\left(V_{1}(\mathbb{A})\right)$ is $\tilde{K}_{H}$-finite and $\varphi$ is $\tilde{K}_{H}^{\prime}$-finite, it follows from Proposition 3.1 that $\theta_{\varphi_{V}}\left(f_{1}\right)$ is an automorphic from on $\left.\tilde{H}(\mathbb{A})\right)$ and $\theta_{\varphi}\left(f_{2}\right)$ is an automorphic from on $\left.\tilde{H}^{\prime}(\mathbb{A})\right)$. Since $\theta_{\varphi_{\nu}, \varphi}^{\tilde{\rho}, \tilde{H}_{H}}(f)=\theta_{\varphi_{v}}\left(f_{1}\right) \cdot\left(\theta_{\varphi}\left(f_{2}\right) \circ \tilde{\rho}_{H}\right)$, the proposition follows from this and Definition 2.3.

\section{Geometric applications}

Families of abelian varieties parametrized by an arithmetic variety play an important role in number theory. In this section we describe some applications of generalized theta-series liftings discussed in Section 3 and Section 4 to such families of abelian 
varieties. Let $\mathbb{G}$ be a semisimple algebraic group over $\mathbb{Q}$ and let $G=\mathbb{G}(\mathbb{R})$ be its group of real points. Let $K$ be a maximal compact subgroup of the semisimple Lie group $G$, and assume that the corresponding symmetric space $D=G / K$ has a $G$ invariant complex structure. Let $W$ be a vector space over $\mathbb{Q}$, and let $\rho: \mathbb{G} \rightarrow S p(W)$ be a homomorphism defined over $\mathbb{Q}$. Let $\mathfrak{G}$ be the Siegel upper half space on which $S p(W)(\mathbb{R})$ operates, and let $\tau: D \rightarrow \mathfrak{h}$ be a holomorphic map that is equivariant with respect to the Lie group homomorhism $\rho_{R}: G \rightarrow S p(W)(\mathbb{R})$ determined by $\rho$, that is, $\tau(g x)=\rho_{R}(g) \tau(x)$ for all $g \in G$ and $x \in D$. If $\Gamma$ is a torsion-free cocompact arithmetic subgroup of $\mathbb{G}(\mathbb{Q})$, then the equivariant pair $\left(\rho_{R}, \tau\right)$ determines a Kuga fiber variety $Y \rightarrow X$ which is a family of abelian varieties parametrized by the arithmetic variety $X_{\Gamma}=\Gamma \backslash D$ (see for example $[7,10,12,13,15]$ ).

EXAMPLE 5.1. Let $\mathbb{G}_{\rho}$ be the centralizer of $\rho(\mathbb{G})$ in $S p(W)$, and let $X_{\rho}$ be the set of holomorphic maps $\tau: D \rightarrow \mathfrak{h}$ satisfying the relation $\tau(g x)=\rho_{R}(g) \tau(x)$ for all $x \in D$. Then $\mathbb{G}_{\rho}(\mathbb{R})$ operates on $X_{\rho}$ by $\gamma \cdot \tau=\gamma \circ \tau$ for $\gamma \in \mathbb{G}_{\rho}(\mathbb{R})$ and $\tau \in X_{\rho}$. Then it is known that this action is transitive and that $X_{\rho}$ is the symmetric space corresponding to the semisimple Lie group $\mathbb{G}_{\rho}(\mathbb{R})$ (see [8]). The pair $\left(\rho(\mathbb{G}), \mathbb{G}_{\rho}\right)$ of subgroups of $S p(W)$ is not quite a dual reductive pair because $\rho(\mathbb{G})$ may be smaller than the centralizer of $\mathbb{G}_{\rho}$. However, as is explained in $[5$, p. 66], the theta correspondence of the form given in Proposition 3.1 still exists as long as the two subgroups commute. Thus Theorem 3.3 essentially suggests that the theta-series lifting associates an automorphic form on $\mathbb{G}_{\rho}(\mathbb{A})$ to each cusp form on $\mathbb{G}(\mathbb{A})$.

In order to consider another application, let $\mathbb{G}^{\prime}, \mathbb{W}^{\prime}$ and $\mathbb{H}$ be reductive groups over $\mathbb{Q}$ such that $\left(\mathbb{G}^{\prime}, \mathbb{W}^{\prime}\right)$ is a dual reductive pair in $S p(W)$ and $(\mathbb{G}, \mathbb{N})$ is a dual reductive pair in another symplectic group $S p(V)$. We also assume that $\rho(\mathbb{G}) \subset \mathbb{G}^{\prime}$ and that there is a homomorphism $\rho_{H}: \mathbb{W} \rightarrow \mathbb{W} \mathbb{N}^{\prime}$ defined over $\mathbb{Q}$.

EXAMPLE 5.2. Let $f_{1}$ be the automorphic form on $\mathbb{G}(\mathbb{A})$ determined by the canonical automorphy factor (see $[15, \S[I .5]$ ) of $\mathbb{G}(\mathbb{C})$ on $D$, and given a positive integer $n$ let $f_{2}$ be the automorphic form on $S p(W)(\mathbb{A})$ determined by the automorphy factor $j: S p(W)(\mathbb{R}) \times \mathfrak{h} \rightarrow \mathbb{C}$ given by

$$
j(g, z)=\operatorname{det}(c z+d)^{n}
$$

for $z \in \mathfrak{h}$ and $g=\left(\begin{array}{ll}a & b \\ c & d\end{array}\right) \in S p(W)(\mathbb{R})$. Then it is known that the space of mixed cusp forms on $\mathbb{G}(\mathbb{A})$ of the form $f_{1} \cdot\left(f_{2} \circ \rho\right)$ is canonically isomorphic to the space of holomorphic forms of the highest degree on the $n$th fiber power $Y_{\rho, \tau}^{n}$ of $Y_{\rho, \tau}$ over the arithmetic variety $X_{\Gamma}=\Gamma \backslash D$ (see [13]). Thus using Theorem 4.2 we can associate a mixed automorphic form on $\mathbb{H}(\mathbb{A})$ to each such holomorphic form on $Y_{\rho, \tau}^{n}$. 


\section{References}

[1] A. Borel and H. Jacquet, 'Automorhic forms and automorphic representations', in: Proc. Symp. Pure Math. 33 (Amer. Math. Soc., Providence, 1979) pp. 189-202.

[2] D. Cox and S. Zucker, 'Intersection numbers of sections of elliptic surfaces', Invent. Math. 53 (1979), 1-44.

[3] S. Gelbart, 'On theta series liftings for unitary groups', in: Theta functions from the classical to the modern (ed. M. Murty) (Amer. Math. Soc., Providence, 1993) pp. 129-174.

[4] R. Howe, ' $\theta$-series and invariant theory', in: Proc. Symp. Pure Math. 33 (Amer. Math. Soc., Providence, 1979) pp. 275-286.

[5] R. Howe and I. Piatetski-Shapiro, 'Some examples of automorphic forms on $S p_{4}$ ', Duke Math. J. 50 (1983), 55-106.

[6] B. Hunt and W. Meyer, 'Mixed automorphic forms and invariants of elliptic surfaces', Math. Ann. 271 (1985), 53-80.

[7] M. Kuga, Fiber varieties over a symmetric space whose fibers are abelian varieties, I, II (Lecture Notes, Univ. Chicago, 1963/64).

[8] M. Kuga and S.Ihara, 'Family of families of abelian varieties', in: Algebraic number theory (Japan Soc. Prom. Soc., Tokyo, 1977) pp. 129-142.

[9] M. H. Lee, 'Mixed cusp forms and holomorphic forms on elliptic varieties', Pacific J. Math. 132 (1988), 363-370.

[10] _ 'Conjugates of equivariant holomorphic maps of symmetric domains', Pacific J. Math. 149 (1991), 127-144.

[11] _ - 'Mixed cusp forms and Poincaré series', Rocky Mountain J. Math. 23 (1993), 1009-1022.

[12] ____. 'Mixed Siegel modular forms and Kuga fiber varieties', Illinois J. Math. 38 (1994), 692700 .

[13] _ _ 'Mixed automorphic vector bundles on Shimura varieties', Pacific J. Math. 173 (1996), 105-126.

[14] D. Prasad, 'Weil representation, Howe duality, and the theta correspondence', in: Theta functions from the classical to the modern (ed. M. Murty) (Amer. Math. Soc., Providence, 1993) pp. 105-127.

[15] I. Satake, Algebraic structures of symmetric domains (Princeton Univ. Press, Princeton, 1980).

[16] P. Stiller, Special values of Dirichlet series, monodromy, and the periods of automorphic forms, Mem. Amer. Math. Soc. 299 (Amer. Math. Soc., Providence, 1984).

[17] A. Weil, 'Sur certains groupes d'operateurs unitaires', Acta Math. 111 (1964), 143-211.

\section{Department of Mathematics \\ University of Northern Iowa \\ Cedar Falls \\ Iowa 50614 \\ USA}

e-mail: lee@math.uni.edu 